\title{
Clinical significance of early treatment of overjet is questionable
}

\author{
At what age should orthodontic treatment for prominent upper teeth be \\ carried out?
}

\author{
Harrison JE, O'Brien KD, Worthington HV. \\ Orthodontic treatment for prominent upper front teeth in children. \\ Cochrane Database Syst Rev 2007; issue 3
}

Data sources Literature searches were made within the Cochrane Oral Health Group's Trials Register, Cochrane Central Register of Controlled Trials, Medline and Embase, and articles were also identified as part of the Cochrane Oral Health Group's handsearching programme from the following sources: American Journal of Orthodontics and Dentofacial Orthopedics, The Angle Orthodontist, European Journal of Orthodontics and Journal of Orthodontics. In addition, the journals Seminars in Orthodontics, Clinical Orthodontics and Research and Australian Journal of Orthodontics were searched by hand. Bibliographies of identified trials were checked and first-named authors of all trial reports were contacted in an attempt to identify unpublished studies and to obtain any further information about the trials. There were no language restrictions.

Study selection Randomised and controlled clinical trials were chosen if participants were children and/ or adolescents (age $<16$ years) who were receiving orthodontic treatment to correct prominent upper front teeth using any orthodontic brace or head-brace, and where the control was no or delayed treatment or another active intervention. Primary outcomes considered were prominence of the upper front teeth, relationship between upper and lower jaws. Secondary outcomes were self esteem, any injury to the upper front teeth, jaw joint problems, patient satisfaction, and the number of attendances required to complete treatment.

Data extraction and synthesis Information regarding methods, participants, interventions, outcome measures and results were extracted independently and in duplicate by two review authors. The Cochrane Oral Health Group's statistical guidelines were followed and mean differences were calculated using random-effects models. Potential sources of heterogeneity were examined.
Results Eight trials, based on data from 592 patients who presented with Class II Division 1 malocclusion, were included in the review. Within these, three trials (432 participants) considered early treatment, comparing a functional appliance with no treatment. There was a significant difference in final overjet in the treatment group versus control group of $-4.04 \mathrm{~mm}$ [95\% confidence interval $(\mathrm{Cl}),-7.47--0.60$; $X^{2} 117.02 ; 2$ degrees freedom (df); $\left.\left.P<0.00001, P 298.3 \%\right)\right]$. There was a significant difference in ANB $(-1.35 \mathrm{~mm} ; 95 \% \mathrm{Cl}, 2.57-0.14$; $\left.X^{2} 9.17 ; 2 \mathrm{df} ; P 0.01 ; P^{2} 78.2 \%\right)$ and change in ANB $(-0.55 ; 95 \% \mathrm{Cl}$, $\left.-0.92-0.18 ; X^{2} 5.71 ; 1 \mathrm{df} ; P 0.06 ; R^{2} 65.0 \%\right)$ between treatment and control groups. The comparison of the effect of treatment with headgear versus an untreated control revealed that there was a small but significant effect of headgear treatment on overjet of $-1.07(95 \% \mathrm{Cl}$, $\left.-1.63-0.51 ; X^{2} 0.05 ; 1 \mathrm{df} ; P 0.82 ; I^{2} 0 \%\right)$. Similarly, headgear resulted in a significant reduction in final ANB of $-0.72(95 \% \mathrm{Cl},-1.18--0.27$; $\left.X^{2} 0.34 ; 1 \mathrm{df} ; P 0.56 ; I^{2} 0 \%\right)$. No significant differences, with respect to final overjet, ANB or ANB change, were found between early treatment with headgear and the functional appliances. For trials considering adolescent treatment (phase II), at the end of all treatment there was found to be no significant difference between overjet, final ANB or PAR score in participants who had a course of early treatment with headgear or a functional appliance and in those who had not received early treatment. Similarly, there were no significant differences in overjet, final ANB or PAR score between children who had received a course of early treatment with headgear or a functional appliance. One trial found a significant reduction in overjet $(-5.22 \mathrm{~mm} ; 95 \% \mathrm{Cl},-6.51--3.93)$ and ANB (-2.27 degrees; $95 \% \mathrm{Cl},-3.22-1.31 ; X^{2} 1.9 ; 1 \mathrm{df} ; P 0.17$; $R^{2} 47.3 \%$ ) for adolescents receiving one-phase treatment with a functional appliance versus an untreated control. There was a statistically significant reduction of ANB ( -0.68 degrees; $95 \% \mathrm{Cl},-1.32-0.04$; $\left.X^{2} 0.56 ; 1 \mathrm{df} ; P 0.46 ; I^{2} 0 \%\right)$ with the twinblock appliance compared with other functional appliances, but there was no significant effect of the type of appliance on the final overjet.

Conclusions The evidence suggests that providing early orthodontic treatment for children with prominent upper front teeth is no more effective than providing one course of orthodontic treatment when the child is in early adolescence.

\section{Commentary}

The objective of this Cochrane systematic review (SR) was to evaluate the effect of early or late treatment for upper prominent front teeth. As expected from a Cochrane SR, this is well designed and executed. Only a couple of points warrant further consideration.

Although several electronic databases were searched, there is another that may have an impact on the results. Lilacs, the LatinoAmerican and Caribbean Health Sciences Literature database, is not covered at all by any of the databases utilised here. It includes Spanish and Portuguese published literature, however, and high levels of dental-related research have been noted lately in Brazil: work
Address for correspondence: Luisa Fernandez, Group Co-ordinator,

University of Manchester, Higher Cambridge Street, Manchester M15 6FH, UK.

E-mail luisa.fernandez@manchester.ac.uk 
from all three countries may have provided some useful references. Some initial reports show that not including this database may have some impact in the results of SR. ${ }^{1}$

All the identified and selected clinical trials analysed dental and skeletal effects with or without treatment. It became clear that even if an early intervention does show a statistically significant difference, the magnitude of the change is probably not clinically significant enough on its own to justify the intervention. Outcome measures not included that would be of clinical significance are the psychological impact of the intervention and the number of accidents involving the protruding front teeth. Future studies should consider such variables.

A final point to be emphasised, which is noted in this review, is that fragmentation of research reports only adds confusion to any conclusions that can be drawn. As the authors suggest, clinical trial reports should only be published when completed and not in a fragmented version.

\section{Practice point}

There is evidence that early treatment reduces overjet associated with protruding frontal teeth. The clinical significance of the reduction is questionable.

Important outcome measurements (psychological impact and reduction of associated accidents) have not been evaluated in the selected literature but are important if deciding on treatment to reduce early incisor protrusion.

\section{Carlos Flores-Mir}

Craniofacial and Oral-health Evidence-based Practice Group, Faculty of Medicine and Dentistry University of Alberta, Edmonton, Alberta, USA

1. Clark OA, Castro AA. Searching the Literatura Latino Americana e do Caribe em Ciencias da Saude (LILACS) database improves systematic reviews. Int J Epidemiol. 2002 Feb;31(1):112-4

Evidence-Based Dentistry (2007) 8, 103-104. doi:10.1038/sj.ebd.6400523 